\title{
Evidence for Treatment and Survival Disparities by Age in Pancreatic Adenocarcinoma:
}

\section{A Population-Based Analysis}

\author{
Sunil Amin, MD, $\mathrm{MPH}^{\star}, \dagger$, Aimee L. Lucas, MS, MD ${ }^{\dagger, \ddagger}$, and Harold Frucht, MD ${ }^{\dagger, \ddagger}$ \\ *Department of Internal Medicine, Mount Sinai School of Medicine, New York, NY \\ †Muzzi Mirza Pancreatic Cancer Prevention \& Genetics Program, New York, NY \\ ¥Division of Digestive and Liver Diseases, Department of Medicine, Columbia University College \\ of Physicians and Surgeons, New York, NY
}

\begin{abstract}
Objectives-Studies demonstrate safety and survival benefits of surgical resection in older individuals with pancreatic adenocarcinoma. We investigated treatment disparities by age.
\end{abstract}

\begin{abstract}
Methods-The Surveillance, Epidemiology, and End Results database for survival and treatment of pancreatic adenocarcinoma between 1983 and 2007 stratified by age: younger than 50 years, between 50 and 70 years, or older than 70 years. Kaplan-Meier curves and Cox proportional hazards models were used for survival differences, and logistic regression models were used for treatment disparities and the decision to refuse surgery.
\end{abstract}

Results-A total of 45,509 patients had microscopically confirmed pancreatic adenocarcinoma. Of these, $7374(16 \%)$ received surgery and $9842(22 \%)$ received radiation. Younger patients were more likely to receive both surgery and radiation. The prevalence of surgery decreased from $21 \%$ for those younger than 50 years to $19 \%$ for those between 50 and 70 years to $13 \%$ for those older than 70 years $(P<0.001)$. Radiation decreased from $28 \%$ to $25 \%$ to $17 \%(P<0.001)$. Overall survival decreased with increasing age at diagnosis, 10.4 months (age $<50$ years) to 9.1 months (age 50-70 years) to 6.4 months (age $>70$ years) controlling for stage, sex, race, radiation, and surgery $(P<0.001)$. Increasing age negatively predicted the odds of receiving both surgery and radiation and increased the likelihood of refusing surgery.

Conclusions-Treatment disparities exist by age despite advances in radiation and surgical treatment. Increased treatment in the elderly will increase overall survival from pancreatic adenocarcinoma.

\section{Keywords}

pancreatic adenocarcinoma; SEER (Surveillance Epidemiology and End Results); epidemiology; age; disparities; geriatrics

Five-year survival rates from pancreatic cancer remain dismal and have changed little over the past 35 years. The current rate of $6 \%$ is only slightly improved from the $3 \%$ documented from 1975 to $1977 .{ }^{1}$ Although pancreatic cancer rarely presents before age 45 , the incidence rises sharply thereafter. As survival from other cancers and chronic diseases improves, the

Copyright ( 92013 by Lippincott Williams \& Wilkins

Reprints: Harold Frucht, MD, 630 W 168th St, Box No. 83, New York City, NY 10032 (hf2011@ columbia.edu).

The authors declare no conflict of interest. 
number of elderly patients diagnosed with pancreatic cancer is increasing. As such, age is an important risk factor in the development of pancreas cancer. Whereas the overall incidence rate of pancreatic cancer for all ages is 11.7 per 100,000, the incidence rate for individuals older than 65 years is 66.4 per 100,000. Among adults older than 80 years, the incidence rate is as high as 91.1 per $100,000 .^{2}$

Surgical resection remains the only potential cure for patients with localized pancreatic cancer, and studies confirm the safety and the survival benefit of surgical resection in older individuals. ${ }^{3-6}$ Most recently, Lee et al ${ }^{7}$ and Stauffer et al ${ }^{8}$ demonstrated that pancreaticoduodenectomy can be performed safely in patients 80 years and older. Despite these reports, many surgeons are wary of performing surgical resection with intent to cure for octogenarians-a particularly troubling trend given the lack of efficacious alternate treatment modalities.

For locally advanced pancreatic cancer, chemoradiation is the standard of care. ${ }^{9,10}$ However, when such aggressive regimens are considered, elderly patients may again be at a disadvantage as a result of poor health or the fear of aggressive management in this population. However, individuals 75 years or older have similar outcomes in terms of overall survival after chemoradiation compared with the much younger historic controls (mean age, 59-62 years) reported in prospective randomized trials. ${ }^{11}$ Nevertheless, similar to surgery, medical and radiation oncologists remain reluctant to pursue such regimens in older patients, as evidenced by data from the SEER Medicare database. ${ }^{12}$ With regard to metastatic pancreatic cancer, single-agent gemcitabine has long been the treatment of choice, with little progress made with regard to combination therapies. ${ }^{13}$ Recently, however, combination chemotherapy with oxaliplatin, irinotecan, fluorouracil, and leucovorin (FOLFIRINOX) has shown promise among younger patients with good functional status (median age, 61 years). ${ }^{14}$ Unfortunately, a lack of evidence for use in older individuals combined with a higher incidence of adverse events (febrile neutropenia, thrombocytopenia, diarrhea, and sensory neuropathy) compared with gemicitibine alone may further bias oncologists from offering this or other regimens to older patients.

As the US population ages and the management of pancreatic cancer patients diagnosed after age 75 becomes more common, decisions regarding aggressive care will become increasingly relevant. We used a population-based database to quantify treatment disparities by age and whether they are changing over time. We hypothesize that treatment disparities by age, despite advances in both medical and surgical technology, may partly explain the persistently high 5-year mortality associated with pancreatic cancer.

\section{Materials and Methods}

The National Cancer Institute's Surveillance, Epidemiology, and End Results (SEER) database was used to perform a retrospective review of survival and treatment data for patients diagnosed with pancreatic adenocarcinoma between 1983 and 2007. ${ }^{15}$ SEER*Stat software ${ }^{16}$ was used to initially select patients with pancreatic tumors based on site codes C250-C254 and C257-C259. The International Classification of Diseases for Oncology, 3rd Edition, histology codes 8140-8141, 8143, 8147, 8210-8211, 8260-8263, 8440, 8503, 8560 , and $8570-8576$ were then used to select for pancreatic adenocarcinoma specifically. We included all pancreatic adenocarcinomas that were microscopically confirmed, occurred in persons older than 25 years, and were listed as the patient's principal cause of death. Exclusion criteria included cancer only noted on death certificate or autopsy and cancers for which treatment with radiation and/or surgery was unknown. Patients diagnosed before 1983 were ineligible given the lack of surgical data before this date in the SEER database. 
Patients were stratified into groups based on age at diagnosis $(<50,50-70$, or $>70$ years $)$ and year of diagnosis (1983-1990, 1991-2000, and 2001-2007).

Kaplan-Meier curves were constructed, and the log-rank test was used to test survival differences between age strata. Cox proportional hazards models were then constructed to control for additional variables. Treatment disparities for radiation and surgery by age strata were assessed first with $\chi^{2}$ tests and then by multivariate logistic regression models.

Treatment disparities for chemotherapy could not be assessed because this information is not readily available in the SEER database. Statistical significance was accepted at the $P<0.05$ level. All statistical analyses were performed using SAS v9.2 (SAS Institute, Cary, NC).

\section{Results}

We identified 45,509 patients with microscopically confirmed pancreatic adenocarcinoma diagnosed between 1983 and 2007 (Table 1). Most (26,220, 58\%) of these cases occurred between 2001 and 2007. Of these patients, 51\% were male and $82 \%$ self-identified as white. Mean (SD) age at diagnosis was 67.8 (11.6) years. Of all patients, 3267 (7\%) had localized disease, 14,641 (32\%) had regional disease, and 27,601 (61\%) had distant disease. Stratified by age, there was an increasing prevalence of localized (ie, resectable) lesions among older patients (5\% [ $<50$ years] vs 6\% [50-70 years] vs $9 \%$ [ $>70$ years], $P<0.001)$. During the study period (1983-2007), 7374 patients (16\%) received surgery and 9842 patients (22\%) received radiation treatment (Table 2).

\section{Treatment Disparities By Age}

When stratified by age, younger patients were more likely to receive both surgery and radiation. The rate of surgery decreased from $21 \%$ for those younger than 50 years to $19 \%$ for those between 50 and 70 years to $13 \%$ for those older than 70 years $\left(\chi^{2}=364, P<\right.$ 0.001 ). The rate of radiation decreased from $28 \%$ to $25 \%$ to $17 \%$ for the same age groups $\left(\chi^{2}=541, P<0.001\right)$. In the multivariate analysis, increasing age negatively predicted the odds of receiving both surgery and radiation. This disparity did not lessen over time (Table 3). Compared with patients younger than 50 years, the odds of receiving surgery for those 50 to 70 years old was $17 \%$ less from 1983 to $1990,23 \%$ less from 1991 to 2000 , and $22 \%$ less from 2001 to 2007. For patients older than 70 years, the odds of surgery were $55 \%$ less, $57 \%$ less, and $60 \%$ less during the same periods. Similarly, the odds of receiving radiation for patients 50 to 70 years old compared with those younger than 50 years was $26 \%$ less between 1983 and 1990, 19\% less between 1991 and 2000, and 22\% less between 2001 and 2007. For patients older than 70 years, the odds of radiation was $61 \%$ less, $58 \%$ less, and $60 \%$ less.

\section{Patient Refusal of Surgery by Age}

Older patients were also more likely to refuse surgical resection of pancreatic cancer. In a separate analysis of 47,374 patients from the SEER database diagnosed with histologically confirmed pancreatic adenocarcinoma from 1983 to 2007, those 477 patients who were recommended to undergo cancer-directed surgery but refused had a mean (SD) age of 73.9 (10.5) years compared with 67.7 (11.6) years for the entire cohort $(P<0.01)$. On both univariate and multivariate analyses, factors predictive of the decision to refuse surgery were increasing age (odds ratio [OR], 1.713 [95\% confidence interval $\{\mathrm{CI}\}, 1.012-2.900 ; P$ $=0.045]$ for 50-70 years versus <50 years; OR, 5.712 [95\% CI, 3.423-9.533; $P<0.001$ ] for $>70$ years versus $<50$ years), black race (OR, 1.527 [95\% CI, 1.141-2.042; $P=0.004])$, and being unmarried (OR, 1.824 [95\% CI, 1.493-2.229; $P<0.001]$ ), controlling for stage, year of diagnosis, and urban living. Patients who refused recommended surgery had significantly worse mean survival than those who underwent surgery ( 6.2 vs 16.7 months) both by 
Kaplan-Meier methods $(P<0.001)$ and Cox proportional hazards models (hazard ratio [HR], 2.314 [95\% CI, 2.096-2.554; $P<0.001$ ]) controlling for age, year of diagnosis, race, sex, stage, and marital status.

\section{Survival Disparities by Age}

Overall survival significantly decreased with increasing age at diagnosis, from a mean of 10.4 months for those younger than 50 to 9.1 months for those 50 to 70 years to 6.4 months for those older than 70 years (Kaplan-Maier, $P<0.0001$; Cox proportional hazards models: HR, 1.131 [95\% CI, 1.090-1.174; $P<0.0001$ ] for those 50-70 years vs $<50$ years; HR, 1.485 [95\% CI, $1.430-1.542 ; P<0.0001]$ for those $>70$ years versus $<50$ years), controlling for stage, sex, race, radiation, and surgery (Fig. 1).

\section{Discussion}

As the US population ages and the number of healthy, functional patients diagnosed with cancer after age 75 increases, physicians are increasingly required to make treatment decisions for which no guidelines exist. Our study adds pancreatic adenocarcinoma to an increasing list of cancers, including lung, breast, and cervical, for which treatment disparities and survival differences exist by age. ${ }^{17-20}$ Despite convincing evidence that pancreaticoduodenectomy can be performed safely in individuals as old as 80 years, ${ }^{3-8}$ surgeons often hesitate before proceeding with surgical resection for cure in this demographic. Our population-based analysis shows that despite advancing surgical and medical technologies with resultant lower morbidity and mortality, treatment and survival disparities for pancreatic adenocarcinoma exist by age and are not decreased over time.

A number of factors may influence a surgeon's decision to operate or an oncologist's decision to refer for surgery. Older patients are more prone to polypharmacy, cardiac and pulmonary disease requiring intensive intraoperative monitoring, decreased functional status, risk of diabetes mellitus, and poor nutritional status. Whereas the actuarial life expectancy of a 80 -year-old man is 7.9 years, that of a 60 -year-old man is 20.9 years. ${ }^{21}$ Nevertheless, a diagnosis of pancreatic adenocarcinoma will shorten both men's remaining life expectancy to a similar degree based on our data-3.62\% of expected for an 80-year-old man and $6.75 \%$ of expected for a 60 -year-old man. Further complicating the decision to operate, multivariate analyses of population-based databases have shown age to be an independent predictor of postoperative morbidity and mortality. ${ }^{22,23}$ Nevertheless, with the emergence of dedicated high-volume centers, overall perioperative mortality after pancreatic resection has fallen sharply over the last 3 decades and is now less than $3 \% .{ }^{24,25}$ Unfortunately, overall survival from pancreatic cancer has not kept pace, having increased from just $3 \%$ to $6 \%$ during the same period. ${ }^{1}$

One explanation for this inconsistency is that fewer older patients are treated at dedicated high-volume centers. Riall et al ${ }^{26}$ showed that, in Texas, $63 \%$ of patients younger than 60 years were treated at dedicated centers, but this percentage fell gradually to $62 \%$ for patients aged 60 to 69 years, $57 \%$ for patients aged 70 to 79 years, and $54 \%$ for patients 80 years and older $(P<0.01)$. This disparity is particularly relevant because the same study showed that the difference in perioperative mortality between high- and low-volume centers is augmented with increasing age group. Among patients younger than 60 years, only a $1 \%$ mortality difference was observed between high- and low-volume centers (2\% vs $3 \%$ ). However, among patients 80 years and older, this difference in perioperative mortality grew to almost $6 \%(8.7 \%$ vs $14.5 \%)$.

Our population-based study of 45,509 pancreatic adenocarcinoma patients in the SEER database provides further evidence for survival disparities by age, but also specifically looks 
at trends over time as well as the decision to refuse recommended surgery. Our multivariate regression models highlight 2 important observations. First, we show that increasing age negatively predicts both the odds of receiving surgery and radiation treatment regardless of year of diagnosis. Perhaps more importantly, this disparity is not lessening over time despite improvements in therapy. Whereas a patient older than 70 years had a $55 \%$ less odds of receiving surgery than a patient younger than 50 years from 1983 to 1990, the same patient would have a $70 \%$ less odds of receiving the same surgery 10 years later (controlling for stage, age, sex, and race). Similarly, with regard to radiotherapy, the odds of a patient older than 70 years compared with a patient younger than 50 years receiving treatment has remained between 58\% and 61\% less over 3 decades. Second, our subgroup analysis of 477 patients who refused surgery suggests that older individuals are more likely to refuse recommended surgical resection controlling for stage, sex, marital status, race, and urban living. We also demonstrate that patients who refuse recommended surgery have a worse prognosis than those who do not. A lack of understanding regarding the safety of surgery in the older demographic as well as differing perceptions regarding the risks and benefits of surgery among the elderly likely contributes to this observation. As such, we hypothesize that this continued disparity in access to care over time, despite advances in surgery and chemoradiation, coupled with both an increasing diagnosis rate in the elderly and an increased likelihood among this population to refuse surgery may in part explain the persistently dismal overall survival from pancreatic cancer.

Our study is not without limitations. First, working within the limitations of the SEER database, we were unable to control for the various comorbidities or functional status of our study participants. Presumably, patients diagnosed at a later age have more comorbidities and worse functional status that may affect the decision to offer surgery or chemoradiation. Nevertheless, these factors are unlikely to explain all of the variation in odds that we observed, and analysis of the SEER Medicare database controlling for comorbidities has shown an insignificant difference in mortality after pancreatic resection between patients 65 to 69 years and patients older than 80 years. ${ }^{27}$ Second, our study has a clear selection bias. As a retrospective study, we do not know exactly how patients were selected to receive surgery or radiation. However, major factors driving the decision to offer care likely remain constant over time. Finally, we were unable to stratify our data based on the type of center (ie, high vs low volume) at which particular patients were diagnosed. We simply report national trends that likely vary based on region and center.

In conclusion, these data show that recent advances in surgical technology and chemoradiation have not carried over to benefit individuals diagnosed with pancreatic adenocarcinoma at an older age. Despite a sharp reduction in perioperative mortality after resection, the 5-year survival from pancreatic cancer has increased only $3 \%$ in 3 decades. We hypothesize that the persistent treatment disparities over time between age groups combined with the increasing proportion of patients diagnosed after age 70 may partly explain this lack of improvement. Although many factors influence the decision to treat pancreatic adenocarcinoma, age alone should not justify the decision of whether to operate or not or to provide chemoradiation or not.

\section{References}

1. Jemal A, Siegel R, Xu J, et al. Cancer statistics, 2010. CA Cancer J Clin. 2010; 60:277-300. [PubMed: 20610543]

2. Altekruse, SF.; Kosary, CL.; Krapcho, M., et al., editors. SEER Cancer Statistics Review, 19752007. Bethesda, MD: National Cancer Institute; 2010. Available at: http://seer.cancer.gov/csr/ 1975_2007/. Based on November 2009 SEER data submission, posted to the SEER Web site [Accessed December 29, 2011] 
3. Delcore R, Thomas JH, Hermreck AS. Pancreaticoduodenectomy for malignant pancreatic and periampullary neoplasms in elderly patients. Am J Surg. 1991; 162:532-535. [PubMed: 1670220]

4. Hannoun L, Cristophe M, Ribeiro J, et al. A report of forty-four instances of pancreatico- duodenal resection in patients more than seventy years of age. Surg Gynecol Obstet. 1993; 177:556-560. [PubMed: 7903483]

5. Scurtu R, Bachellier P, Oussoultzoglou E, et al. Outcome for pancreaticoduodenectomy for cancer in elderly patients. J Gastrointest Surg. 2006; 10:813-822. [PubMed: 16769537]

6. Oliverius M, Kala Z, Varga M, et al. Radical surgery for pancreatic malignancy in the elderly. Pancreatology. 2010; 10:499-502. [PubMed: 20720452]

7. Lee M, DiNorcia J, Reavey P, et al. Pancreaticoduodenectomy can be performed safely in patients aged 80 years and older. J Gastrointest Surg. 2010; 14:1838-1846. [PubMed: 20824366]

8. Stauffer JA, Grewal MS, Martin JK, et al. Pancreas surgery is safe for octogenarians. J Am Geriatr Soc. 2011; 59:184-186. [PubMed: 21226703]

9. Gastrointestinal Tumor Study Group. Treatment of locally unresectable carcinoma of the pancreas: comparison of combined modality therapy (chemotherapy plus radiotherapy) to chemotherapy alone. J Natl Cancer Inst. 1988; 80:751-755. [PubMed: 2898536]

10. Gastrointestinal Tumor Study Group. A multi-institutional comparative trial of radiation therapy alone and in combination with 5-fluorouracil for locally unresectable pancreatic carcinoma. Ann Surg. 1979; 189:205-208. [PubMed: 426553]

11. Miyamoto DT, Mamon HJ, Ryan DP, et al. Outcomes and tolerability of chemoradiation therapy for pancreatic cancer patients aged 75 years or older. Int J Radiat Oncol Biol Phys. 2010; 77:11711177. [PubMed: 19800182]

12. Krzyzanowska MK, Weeks JC, Earle CC. Treatment of locally advanced pancreatic cancer in the real world: population-based practices and effectiveness. J Clin Oncol. 2003; 21:3409-3414. [PubMed: 12972517]

13. Di Marco M, Di Cicilia R, Macchini M, et al. Metastatic pancreatic cancer: is gemcitabine still the best standard treatment? Oncol Rep. 2010; 23:1183-1192. [PubMed: 20372829]

14. Conroy T, Desseigne F, Ychou M, et al. FOLFIRINOX versus gemcitabine for metastatic pancreatic cancer. N Engl J Med. 2011; 364:1817-1825. [PubMed: 21561347]

15. SEER*Stat Database: Incidence- SEER 9 Regs Limited-Use, Nov 2008 Sub (1973-2006) <Katrina/Rita Population Adjustment> - Linked To County Attributes - Total US, 1969-2006 Counties. Bethesda, MD: National Cancer Institute, DCCPS, Surveillance Research Program, Cancer Statistics Branch; 2009. Surveillance, Epidemiology, and End Results (SEER) Program. Based on the November 2008 submission. Available at: www.seer.cancer.gov [Accessed December 29, 2011]

16. Surveillance Research Program, National Cancer Institute SEER*Stat software. [Accessed December 29, 2011] Available at: www.seer.cancer.gov/seerstat. Version 6.5.2

17. Cykert S, Dilworth-Anderson P, Monroe MH, et al. Factors associated with decisions to undergo surgery among patients with newly diagnosed early-stage lung cancer. JAMA. 2010; 303:23682376. [PubMed: 20551407]

18. Schonberg MA, Marcantonio ER, Li D, et al. Breast cancer among the oldest old; tumor characteristics, treatment choices, and survival. J Clin Oncol. 2010; 29:2038-2045. [PubMed: 20308658]

19. Sharma C, Deutsch I, Horowitz DP, et al. Patterns of care and treatment outcomes for elderly women with cervical cancer. Cancer. 2011; 28 E-pub ahead of print.

20. Samet J, Hunt WC, Key C, et al. Choice of cancer therapy varies with age of patient. JAMA. 1986; 255:3385-3390. [PubMed: 3712698]

21. [Accessed January 22, 2012] Period Life Table, Actuarial Publications. Available at: www.ssa.gov/ oact/STATS/table4c6.html

22. de la Fuente SG, Bennet KM, Pappas TN, et al. Pre- and intraoperative variables affecting early outcomes in elderly patients undergoing pancreaticoduodenectomy. HPB (Oxford). 2011; 13:887892. [PubMed: 22081925]

23. Parikh P, Shiloach M, Cohen ME, et al. Pancreatectomy risk calculator: an ACS-NSQIP resource. HPB (Oxford). 2011; 12:488-497. [PubMed: 20815858] 
24. Sohn TA, Yeo CJ, Cameron JL, et al. Resected adenocarcinoma of the pancreas-616 patients: results, outcomes, and prognostic indicators. J Gastrointest Surg. 2000; 4:567-579. [PubMed: 11307091]

25. Trede M, Schwall G, Saeger HD. Survival after pancreatoduodenectomy. 118 consecutive resections without an operative mortality. Ann Surg. 1990; 211:447-458. [PubMed: 2322039]

26. Riall TS, Reddy DM, Nealon WH, et al. The effect of age on short-term outcomes after pancreatic resection: a population-based study. Ann Surg. 2008; 248:459-467. [PubMed: 18791366]

27. Finlayson E, Fan Z, Birkmeyer JD. Outcomes in octogenarians undergoing high-risk cancer operation: a national study. J Am Coll Surg. 2007; 205:729-734. [PubMed: 18035254] 


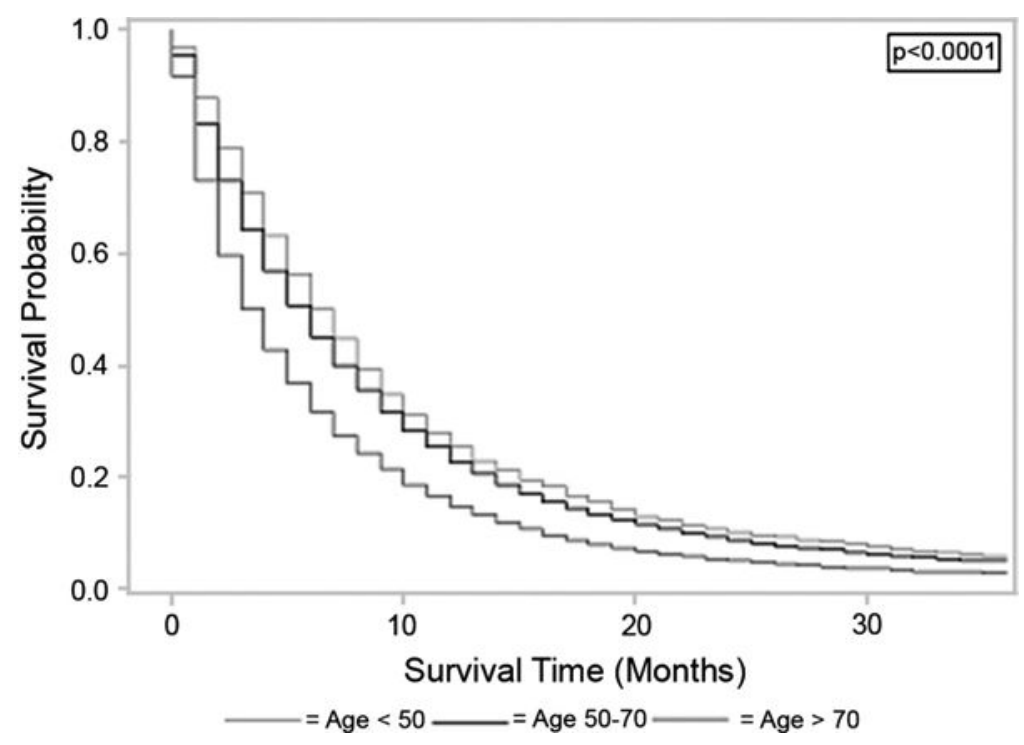

Figure 1.

This figure demonstrates the association between increasing age at diagnosis of pancreatic adenocarcinoma and worse survival. Patients diagnosed between ages 50 to 70 years and after age 70 years have significantly worse survival than those diagnosed before age 50 years, both by Kaplan-Meier methods (log-rank, $P<0.0001)$ and Cox proportional hazards models (HR, 1.131 [95\% CI, 1.090-1.174, $P<0.0001$ ] for 50-70 years vs <50 years; HR, 1.485 [95\% CI, $1.430-1.542, P<0.0001]$ for $>70$ years vs $<50$ years). 
Table 1

Baseline Characteristics of Pancreatic Adenocarcinoma Cases by Age Group, SEER 1983 to 2007

\begin{tabular}{|c|c|c|c|c|}
\hline $\begin{array}{l}\text { Demographic } \\
\text { Characteristic, n (\%) }\end{array}$ & Overall Cohort $(n=45,509)$ & Age $<50$ y $(n=3710)$ & Age 50-70 y $(n=21,446)$ & Age $>70$ y $(n=20,353)$ \\
\hline \multicolumn{5}{|l|}{ Race } \\
\hline White & $37,399(82)$ & 2845 (77) & $17,263(81)$ & $17,291(85)$ \\
\hline African-American & $4969(11)$ & $563(15)$ & $2697(13)$ & $1709(8)$ \\
\hline Other & $3141(7)$ & $302(8)$ & $1486(6)$ & $1353(7)$ \\
\hline \multicolumn{5}{|l|}{ Sex } \\
\hline Male & $23,173(51)$ & $1516(41)$ & $11,798(55)$ & $9181(45)$ \\
\hline Female & $22,336(49)$ & $2194(59)$ & $9648(45)$ & $11,172(55)$ \\
\hline \multicolumn{5}{|l|}{ Year of diagnosis } \\
\hline $1983-1990$ & $3491(8)$ & $280(8)$ & $1828(9)$ & $1383(7)$ \\
\hline $1991-2000$ & $15,798(35)$ & $1323(36)$ & $7446(35)$ & $7029(35)$ \\
\hline $2001-2007$ & $26,220(58)$ & $2107(57)$ & $12,172(57)$ & $11,941(59)$ \\
\hline \multicolumn{5}{|l|}{ SEER historic stage } \\
\hline Localized & $3267(7)$ & $187(5)$ & $1222(6)$ & $1858(9)$ \\
\hline Regional & $14,641(32)$ & $1125(30)$ & $6962(32)$ & $6554(32)$ \\
\hline Distant & $27,601(61)$ & $2398(65)$ & $13,262(62)$ & $11,941(59)$ \\
\hline
\end{tabular}




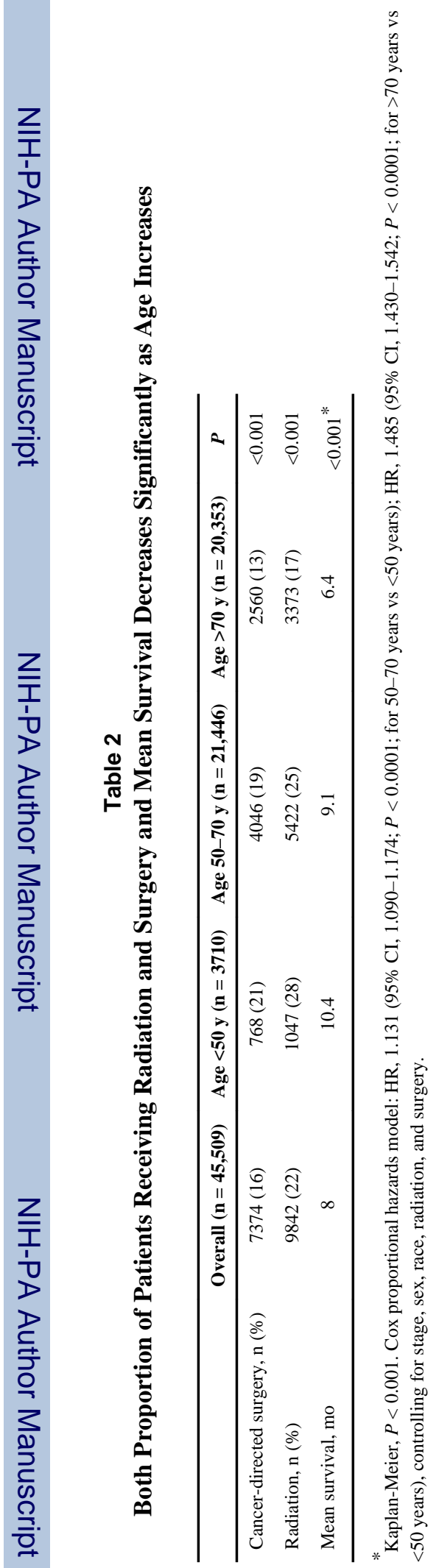

Pancreas. Author manuscript; available in PMC 2014 March 19. 
Table 3

Multivariate Regression Model Predicting Odds of Receiving Surgery or Radiation Over Time

\begin{tabular}{lllll}
\hline & Overall & 1983-1990 & 1991-2000 & 2001-2007 \\
\hline $\begin{array}{l}\text { Odds of cancer-directed surgery } \\
\text { Reference: age }<50 \mathrm{y}\end{array}$ & & & & \\
$\quad$ Age $50-70 \mathrm{y}$ & 0.798 & 0.825 & 0.766 & 0.787 \\
$\quad$ Age $>70 \mathrm{y}$ & 0.421 & 0.451 (NS) & 0.433 & 0.404 \\
$\begin{array}{l}\text { Odds of radiation } \\
\text { Reference: age }<50 \mathrm{y}\end{array}$ & & & & \\
$\quad$ Age $50-70 \mathrm{y}$ & & & & \\
Age $>70 \mathrm{y}$ & 0.792 & 0.739 & 0.812 & 0.777 \\
\hline
\end{tabular}

* All logistic regressions models controlled for stage, age, sex, and race. All $P$ values were significant $(<0.05)$ unless indicated. 